\title{
Bayesian Analysis of Record Statistic from the Inverse Weibull Distribution under Balanced Loss Function
}

\author{
Fuad S. Al-Duais \\ Mathematics Department, College of Humanities and Science in Al-Aflaj, Prince Sattam Bin Abdulaziz University, Al-Kharj, \\ Saudi Arabia \\ Correspondence should be addressed to Fuad S. Al-Duais; f.alduais@psau.edu.sa
}

Received 3 October 2020; Revised 12 January 2021; Accepted 9 March 2021; Published 25 March 2021

Academic Editor: Akif Akgul

Copyright (c) 2021 Fuad S. Al-Duais. This is an open access article distributed under the Creative Commons Attribution License, which permits unrestricted use, distribution, and reproduction in any medium, provided the original work is properly cited.

The main contribution of this work is to develop a linear exponential loss function (LINEX) to estimate the scale parameter and reliability function of the inverse Weibull distribution (IWD) based on lower record values. We do this by merging a weight into LINEX to produce a new loss function called weighted linear exponential loss function (WLINEX). We then use WLINEX to derive the scale parameter and reliability function of the IWD. Subsequently, we discuss the balanced loss functions for three different types of loss function, which include squared error (SE), LINEX, and WLINEX. The majority of previous scholars determined the weighted balanced coefficients without mathematical justification. One of the main contributions of this work is to utilize nonlinear programming to obtain the optimal values of the weighted coefficients for balanced squared error (BSE), balanced linear exponential (BLINEX), and balanced weighted linear exponential (BWLINEX) loss functions. Furthermore, to examine the performance of the proposed methods-WLINEX and BWLINEX - we conduct a Monte Carlo simulation. The comparison is between the proposed methods and other methods including maximum likelihood estimation, SE loss function, LINEX, BSE, and BLINEX. The results of simulation show that the proposed models BWLINEX and WLINEX in this work have the best performance in estimating scale parameter and reliability, respectively, according to the smallest values of mean SE. This result means that the proposed approach is promising and can be applied in a real environment.

\section{Introduction}

Statistics related to record values are of interest for many real-life applications. For instance, they allow prediction of the possible time of earthquakes, floods, extreme weather events, and life-testing studies. Many scholars have researched record values and associated statistics, including generalized extreme value distribution [1], Lomax distribution [2], Weibull distribution [3]; Yang et al [4, 5], lognormal distribution [6], ratio of Weibull random variables [7], power Lindley model [8], exponential distribution [9], generalized Rayleigh model [10], and inverse Weibull distribution (IWD) [11-16]. In this work, we will investigate IWD based on record values.

IWD is one of the most widely used probability distributions with many real environment applications. This refers to the ability of IWD to model a variety of failure characteristics, such as wear-out periods, useful life, infant mortality, and engineering discipline. The probability density function (PDF) and cumulative distribution function (CDF) of IWD are given as follows, respectively:

$$
\begin{gathered}
f(x)=\lambda \theta \exp \left[-\lambda x^{-\theta}\right], \quad x \geq 0 \lambda, \theta>0, \\
F(x)=\exp \left[-\lambda x^{-\theta}\right], \quad x \geq 0, \lambda, \theta>0 .
\end{gathered}
$$

The reliability function is given as follows:

$$
R(t)=1-\exp \left[-\lambda t^{-\theta}\right], \quad t \geq 0, \lambda, \theta>0 .
$$

Here, $\lambda$ and $\theta$ are scale and shape parameters, respectively.

To estimate the parameters and reliability of IWD, scholars use many approaches including Bayesian and nonBayesian. Many researchers attempt to estimate parameters 
and reliability depending on squared error (SE) loss function. The main criticism of this approach is that SE gives overestimation and underestimation equal importance. Thus, an alternative loss function is needed. One of these alternatives is the linear exponential (LINEX) loss function, which many authors have discussed, including Calabria and Pulcini [17], Gencer and Saraçoğlu [18], Khatun and Matin [19], and Parsian and Kirmani [20].

A new type of loss function called balanced loss function appeared with the aim to utilize the positive criteria of two methods. Balanced loss functions habitually consist of the sum of two estimation methods with different weights. These include balanced squared error (BSE) loss function and balanced linear exponential (BLINEX) loss function [21-23].

\section{Methodology}

The weighted coefficients $\left(\omega_{1}\right.$ and $\left.\omega_{2}\right)$ in balanced loss functions are routinely determined by arbitrary choice without depending upon any mathematical justification. This motivated us to propose a justifiable mathematical approach to determine these coefficients. The proposed approach is the nonlinear programming by minimizing the mean square error (MSE) function with conditions related to weighted coefficients.

Furthermore, we developed a new loss function, which we named weighted linear exponential (WLINEX), by weighting LINEX. We then derived scale parameter and reliability function of the IWD depending on WLINEX. In addition, we employed WLINEX to produce the balanced weighted linear exponential loss function (BWLINEX).

2.1. Record Values and Maximum Likelihood Estimation. Let $X_{1}, X_{2}, X_{3}, \ldots$ be a sequence of independent and identically distributed random variables with CDF $F(x)$ and PDF $f(x)$. Set $Y_{n}=\min \left(X_{1}, X_{2}, X_{3}, \ldots, X_{n}\right), n \geq 1$, and say that $X_{j}$ is a lower record and denoted by $X_{L(i)}$ if $Y_{j}<Y_{j-1}, j>1$. Suppose we observe the first $n$ lower record values $X_{L(1)}, X_{L(2)}, X_{L(3)}, \ldots, X_{L(n)}$ from the IWD whose PDF and CDF are given by (1) and (2), respectively. Based on those lower record values, we have the joint density function of the first $n$ lower record values $X \equiv\left(x_{L(1)}, x_{L(2)}, x_{L(3)}\right.$, $\left.\ldots \ldots, x_{L(n)}\right)$ as given by Sultan [24]:

$$
\begin{aligned}
& f_{1,2,3, \ldots \ldots, n}\left(x_{L(1)}, x_{L(2)}, x_{L(3)}, \ldots \ldots, x_{L(n)}\right) \\
& =f\left(x_{L(n)}\right) \prod_{i=1}^{n-1} \frac{f\left(x_{L(i)}\right)}{1-F\left(x_{L(i)}\right)}, \\
& \quad 0 \leq x_{L(1)}<x_{L(2)}<x_{L(3)}, \ldots \ldots<x_{L(n)}<\infty .
\end{aligned}
$$

Here, $f($.$) and F($.$) are given by (1) and (2), respectively,$ after replacing $x$ by $x_{L(i)}$. The likelihood function based on the $n$ lower record values $x$ is given as follows:

$$
\ell(\lambda, \theta \mid X)=(\lambda \theta)^{n} u \exp \left[-\lambda T_{m}\right], \quad u=\prod_{i=1}^{n} x_{L(i)}^{-\theta-1}, T_{m}=x_{L(n)}^{-\theta} .
$$

We obtain that the log-likelihood function may be written as follows:

$$
L(\lambda, \theta \mid X)=\ln (\ell)=n \ln (\lambda)+n \ln (\theta)-\lambda T_{m}-(\theta+1) \sum_{i=1}^{n} \ln \left(x_{L(i)}\right) .
$$

Assuming that the shape parameter $\theta$ is known, using equation (6), the maximum likelihood estimator (MLE) $\widehat{\lambda}_{\mathrm{ML}}$ of the scale parameter $\lambda$ can be shown to be of the following form:

$$
\widehat{\lambda}_{\mathrm{ML}}=\frac{n}{x_{L(n)}^{-\theta}}
$$

If $\lambda$ is replaced by $\hat{\lambda}_{\mathrm{ML}}$ in equation (3), we can obtain the MLE of reliability function $\widehat{R}_{\mathrm{ML}}(t)$ of $R(t)$ depending on the invariance property:

$$
\widehat{R}_{\mathrm{ML}}(t)=1-\exp \left[-\widehat{\lambda}_{\mathrm{ML}} t^{-\theta}\right]
$$

2.2. Loss Functions. In the following sections, we present the four main types of loss functions under investigation in this work.

2.2.1. Squared Error Loss Function. The SE loss function can be expressed as

$$
L(\widehat{\varphi}, \varphi)=(\widehat{\varphi}-\varphi)^{2}
$$

The Bayes estimator of $\varphi$ based on SE loss function can be obtained as follows:

$$
\widehat{\varphi}_{\mathrm{SE}}=E_{\pi}(\varphi \mid X)
$$

2.2.2. Linear Exponential Loss Function. Varian [25] introduced the LINEX loss function. LINEX is an asymmetric loss function that can be expressed as

$$
L(\Delta) \propto[\exp [c \Delta]-c \Delta-1], \quad c \neq 0,
$$

where $\Delta=(\widehat{\varphi}-\varphi)$. The sign and magnitude of $c$ reflect the direction and degree of asymmetry, respectively. The Bayes estimator relative to LINEX loss function, denoted by $\widehat{\varphi}_{\text {LINEX }}$, is given as follows:

$$
\widehat{\varphi}_{\text {LINEX }}=-\frac{1}{c} \operatorname{Ln}\left[E_{\varphi} \exp [-c \varphi]\right], \quad c \neq 0,
$$

provided that $E_{\varphi}=\left(e^{-c \varphi}\right)$ exists and is finite, where $E_{\varphi}$ denotes the expected value.

2.2.3. Weighted Linear Exponential Loss Functions. The researcher proposes this loss function depending on WLINEX loss function as follows:

$$
L^{\star}(\Delta)=L^{\star}(\widehat{\varphi}-\varphi) \propto w(\varphi)[[\exp [c \Delta]-c \Delta-1]], \quad c \neq 0 .
$$


Here, $\hat{\varphi}$ represents the estimated parameter that makes the expectation of loss function by equation (13) as small as possible. The value $w(\varphi)$ represents the proposed weighted function, which is equal to the following:

$$
w(\varphi)=\exp [-z \varphi]
$$

Depending on the posterior distribution of the parameter $\varphi$ and using the proposed weighted function as in equation (14), we can attain the estimated weighted Bayes of the parameter $\varphi$ as follows:

$$
\begin{aligned}
E\left[L_{w}^{*}(\widehat{\varphi}, \varphi)\right]= & \int_{\forall \varphi} L_{w}(\widehat{\varphi}, \varphi) f(\varphi \mid X) \mathrm{d} \varphi=\int_{\forall \varphi} w(\varphi)[\exp [c(\widehat{\varphi}-\varphi)]-c(\widehat{\varphi}-\varphi)-1] f(\varphi \mid X) \mathrm{d} \varphi \\
= & \int_{\forall \varphi} \exp [-z \varphi] \exp [c(\widehat{\varphi}-\varphi)] f(\varphi \mid X) \mathrm{d} \varphi-\int_{\forall \varphi} \exp [-z \varphi] c(\widehat{\varphi}-\varphi) f(\varphi \mid X) \mathrm{d} \varphi-\int_{\forall \varphi} \exp [-z \varphi] f(\varphi \mid X) \mathrm{d} \varphi \\
= & \exp [c \widehat{\varphi}] \int_{\forall \varphi} \exp [-\varphi(z+c)] f(\varphi \mid X) \mathrm{d} \varphi-c \widehat{\varphi} \int_{\forall \varphi} \exp [-z \varphi] f(\varphi \mid X) \mathrm{d} \varphi \\
& +c \int_{\forall \varphi} \varphi \exp [-z \varphi] f(\varphi \mid X) \mathrm{d} \varphi-\int_{\forall \varphi} \exp [-z \varphi] f(\varphi \mid X) \mathrm{d} \varphi \\
& \exp [c \widehat{\varphi}] E_{\varphi}(\exp [-\varphi(z+c) \mid X])-c \widehat{\varphi} E_{\varphi}(\exp [-z \varphi \mid X]) \\
& +c E_{\varphi}(\varphi \exp [-z \varphi \mid X])-E_{\varphi}(\exp [-z \varphi \mid X]) .
\end{aligned}
$$

It is known that, to find the value of $\hat{\varphi}$ that minimizes $E L_{w}^{\star}(\widehat{\varphi}, \varphi)$, we have to perform the following two steps:

$$
\begin{aligned}
& \text { (i) }\left(\partial E_{\varphi} L_{w}^{\star}(\widehat{\varphi}, \varphi) / \partial \widehat{\varphi}\right)=0 \\
& \frac{\partial E_{\varphi} L_{w}^{\star}(\widehat{\varphi}, \varphi)}{\partial \widehat{\varphi}}=c \exp [c \widehat{\varphi}] E_{\varphi}(\exp [-\varphi(z+c) \mid X]) \\
& \quad-c E_{\varphi}(\exp [-z \varphi \mid X])=0 .
\end{aligned}
$$

Therefore, we can find the following:

$$
c=\exp [c \hat{\varphi}] E_{\varphi}(\exp [-\varphi(z+c) \mid X])=c E_{\varphi}(\exp [-z \varphi \mid X]) .
$$

Consequently, the Bayesian estimation of the parameter $\varphi$ using WLINEX will be

$$
\widehat{\varphi}_{\text {WLINEX }}=\frac{1}{c} \operatorname{Ln}\left[\frac{E_{\varphi}(\exp [(-z \varphi \mid X)])}{E_{\varphi}(\exp [(-\varphi(z+c) \mid X)])}\right], \quad z+c \neq 0 .
$$

(i) $E_{\varphi}=\left(e^{-z \varphi}\right)$ and $E_{\varphi}=\left(e^{-(z+c) \varphi}\right)$ exist and are finite, where $E_{\varphi}$ denotes the expected value.

(ii ) $\left(\partial^{2} E_{\varphi} L_{w}^{\star}(\widehat{\varphi}, \varphi) / \partial \widehat{\varphi}^{2}\right)>0$ at the minimum value computed by (i):

$$
\frac{\partial^{2} E_{\varphi} L_{w}^{\star}(\widehat{\varphi}, \varphi)}{\partial \widehat{\varphi}^{2}}=\frac{\partial}{\partial \widehat{\varphi}}\left[\frac{\partial E_{\varphi} L_{w}^{\star}(\widehat{\varphi}, \varphi)}{\partial \widehat{\varphi}}\right]=c^{2} \exp [c \widehat{\varphi}] E_{\varphi}[\exp [-\varphi(z+c)]]>0 .
$$

Because $\widehat{\varphi}_{\text {WLINEX }}$ satisfies conditions (i) and (ii), it follows that $\widehat{\varphi}_{\text {WLINEX }}$ is the minimum value. Note that the
WLINEX loss function is a generalization of the LINEX loss function, where LINEX is a special case of WLINEX when $z=0$ in equation (18).

2.2.4. Balanced Loss Function. According to AbdEllah [22], the class of balanced loss function (BLF) can be written in the form

$$
\begin{aligned}
L_{\rho, \omega, \tau_{0}}^{k}(\xi(\varphi), \tau)= & \omega_{1} k(\varphi) \rho\left(\tau_{0}, \tau\right)+\omega_{2} k(\varphi) \rho(\xi(\varphi), \tau), \\
& \omega_{1}+\omega_{2}=1,
\end{aligned}
$$

where $\tau$ represents an estimator of parameter $\xi(\varphi), \tau_{0}$ is a chosen prior estimator of $\xi(\varphi)$ that can be obtained by several methods such as maximum likelihood (ML) or least squares, $\omega_{1}$ and $\omega_{2}$ represent weighted coefficients belonging to $[0,1), \rho\left(\xi \tau_{0}, \tau\right)$ is an arbitrary loss function when $\xi(\varphi)$ is estimated by $\tau$, and $k(\varphi)$ is a suitable positive weight function. In this work, we discuss three types of BLF including BSE loss function, BLINEX loss function, and WBLINEX loss function, which is proposed in this work.

1 Balanced Squared Error Loss Function. BSE loss function is obtained by choosing $\rho\left(\tau_{0}, \tau\right)=(\tau-\xi$ $(\varphi))^{2}$ and $k(\varphi)=1$ in equation $(20)$ as follows:

$$
L_{\omega, \tau_{0}}(\xi(\varphi), \tau)=\omega_{1}\left(\tau-\tau_{0}\right)^{2}+\omega_{2}(\tau-\xi(\varphi))^{2},
$$

The Bayes estimation of $\xi(\varphi)$ under $L_{\omega_{,}, \tau_{0}}(\xi(\varphi), \tau)$ is given by

$$
\tau_{\omega, \xi}(X)=\omega_{1} \tau_{0}(X)+\omega_{2} E(\xi(\varphi) \mid X) .
$$

Note that $S E$ loss function is a special case of BSE loss function when $\omega_{1}=0$. 
2 Balanced Linear Exponential Loss Function. The BLINEX is obtained by choosing $\rho\left(\tau_{0}, \tau\right)=\exp [c(\tau-$ $\xi(\varphi))]-c(\tau-\xi(\varphi))-1$ and $k(\varphi)=1$ in equation $(20)$ as follows:

$$
\begin{aligned}
L_{\omega, \tau_{0}}^{*}(\xi(\varphi), \tau) & =\omega_{1}\left[\exp \left[c\left(\tau-\tau_{0}\right)\right]-c\left(\tau-\tau_{0}\right)-1\right] \\
& +\omega_{2}[\exp [c(\tau-\xi(\varphi))]-c(\tau-\xi(\varphi))-1] .
\end{aligned}
$$

The Bayes estimation $\xi(\varphi)$ under $L_{\omega, \tau_{0}}^{*}(\xi(\varphi), \tau)$ is given by

$\tau_{\omega, \xi}^{*}(X)=-\frac{1}{c} \log \left[\omega_{1} \exp \left[-c \tau_{0}(X)\right]+\omega_{2} E(\exp [-c \xi(\varphi)] \mid X)\right]$.

Note that when $\omega_{1}=0$, then BLINEX is exactly LINEX loss function.

3 Balanced Weighted Linear Exponential (BWLINEX) Loss Function. The BWLINEX is obtained by choosing

$$
\begin{aligned}
\rho\left(\tau_{0}, \tau\right)= & \exp [-z \varphi] \exp [c(\tau-\xi(\varphi))]-c(\tau-\xi(\varphi)) \\
& -1 \text { and } k(\varphi)=1,
\end{aligned}
$$

in equation (20) as follows:

$$
\begin{aligned}
L_{\omega, \tau_{0}}^{* *}(\xi(\varphi), \tau)= & \omega_{1} \exp [-z \varphi]\left[\exp \left[c\left(\tau-\tau_{0}\right)\right]-c\left(\tau-\tau_{0}\right)-1\right] \\
& +\omega_{2} \exp [-z \varphi][\exp [c(\tau-\xi(\varphi))]-c(\tau-\xi(\varphi))-1] .
\end{aligned}
$$

and the Bayes estimation $\xi(\varphi)$ under $L_{\omega, \tau_{0}}^{* *}(\xi(\varphi), \tau)$ is given by

$$
\tau_{\omega, \xi}^{* *}(X)=\frac{1}{c} \log \left[\omega_{1} \exp \left[-c \tau_{0}(X)\right]+\omega_{2} \frac{E(\exp [-z \xi(\varphi)] \mid X)}{E(\exp [-(z+c) \xi(\varphi)] \mid X)}\right] .
$$

Note that when $\omega_{1}=0$, the BWLINEX loss function is exactly WLINEX loss function.

2.3. Bayes Estimation. In this section, we derive Bayes estimates of the scale parameter $\lambda$ and the reliability $R(t)$ of the IWD. We use six different loss functions, including SE, LINEX, WLINEX, BSE, BLINEX, and BWLINEX. Under the assumption that the shape parameter $\theta$ is known, we assume a gamma (conjugate prior) for density for $\lambda$ with parameters $\nu$ and $\eta$ :

$$
g(\lambda)=\frac{\nu^{\eta}}{\Gamma(\eta)} \lambda^{\eta-1} \exp [-\nu \lambda], \quad \lambda, \nu, \eta>0 .
$$

Combining the likelihood function in equation (5) with the prior PDFof $\lambda$ in equation (28), we get the posterior of $\lambda$ as

$$
\begin{aligned}
\pi(\lambda \mid X)= & \frac{L(\lambda, X) g(\lambda)}{\int_{0}^{\infty} L(\lambda, X) g(\lambda) \mathrm{d} \lambda}=\frac{D^{n+\eta}}{\Gamma(n+\eta)} \lambda^{n+\eta-1} \exp [-\lambda D], \\
& \lambda, \nu, \eta>0,
\end{aligned}
$$

where $X=\left(x_{L(1)}, x_{L(2)}, x_{L(3)}, \ldots \ldots, x_{L(n)}\right)$,

$$
D=\left(\nu+T_{m}\right) \text {. }
$$

2.3.1. Estimates Based on Balanced Squared Error Loss Function. Based on BSE and using equation (21), the Bayes estimation of a parameter $\gamma$ (which can be the scale parameter $\lambda$ or the reliability function $R(t))$ is given by

$$
\gamma_{\mathrm{BSE}}=\omega_{1} \widehat{\gamma}_{\mathrm{ML}}+\omega_{2} E(\gamma \mid X),
$$

where $\widehat{\gamma}_{\mathrm{ML}}$ is the ML estimate of $\gamma$ and $E(\gamma \mid X)$ can be obtained using

$$
E(\gamma \mid X)=\int_{0}^{\infty} \gamma \pi(\lambda \mid X) \mathrm{d} \gamma
$$

Note 1. When $\gamma=\lambda$ in equation (31), the Bayes estimation under BSE loss function of $\lambda$ and denoted by $\widehat{\lambda}_{\mathrm{BSE}}$ is given by

$$
\widehat{\lambda}_{\mathrm{BSE}}=\omega_{1} \widehat{\lambda}_{\mathrm{ML}}+\omega_{2} E(\lambda \mid X),
$$

where $\widehat{\lambda}_{\mathrm{ML}}$ is the ML estimate of $\lambda$, which can be obtained using equation (7). $E(\lambda \mid X)$ can be obtained using

$$
E(\lambda \mid X)=\int_{0}^{\infty} \lambda \frac{D^{n+\eta}}{\Gamma(n+\eta)} \lambda^{n+\eta-1} \exp [-\lambda D] \mathrm{d} \lambda=\frac{n+\eta}{D}
$$

and $D$ is given by equation (28).

Note 2. When $\gamma=R(t)$ in equation (29), the Bayes estimation under BSE loss function of $R(t)$ and denoted by $\widehat{R}_{\mathrm{BSE}}(t)$ is given by

$$
\widehat{R}_{\mathrm{BSE}}(t)=\omega_{1} \widehat{R}_{\mathrm{ML}}(t)+\omega_{2} E(R(t) \mid X),
$$

where $\widehat{R}_{\mathrm{ML}}(t)$ is the ML estimate of $R(t)$ and can be obtained using equation (8), and $E(R(t) \mid X)$ can be obtained using the following:

$$
\begin{aligned}
E(R(t) \mid X) & =\int_{0}^{\infty} 1-\exp \left[-\lambda t^{-\theta}\right] \frac{D^{n+\eta}}{\Gamma(n+\eta)} \lambda^{n+\eta-1} \exp [-\lambda D] \mathrm{d} \lambda \\
& =1-\left(\frac{D}{D+t^{-\theta}}\right)^{n+\eta}, \quad t \geq 0 .
\end{aligned}
$$

The main contribution of this work is to use nonlinear programming to find the optimal values of $\omega_{1}$ and $\omega_{2}$ to compute $\widehat{\lambda}_{\mathrm{BSE}}$ and $\widehat{R}_{\mathrm{BSE}}(t)$ in equations (33) and (35), respectively. To achieve this target, we minimize the MSE as follows:

$$
\begin{aligned}
\text { Minimize } & : \operatorname{MSE}\left(\widehat{\gamma}_{\mathrm{BSE}}\right)=E\left(\widehat{\gamma}_{\mathrm{BSE}}-\gamma\right)^{2} \\
& =E\left[\left(\omega_{1} \widehat{\gamma}_{\mathrm{ML}}+\omega_{2} E(\gamma \mid X)-\gamma\right)\right]^{2}
\end{aligned}
$$

subject to $\omega_{1}+\omega_{2}=1,0 \leq \omega_{1}<1,0 \leq \omega_{2}<1$. 
2.3.2. Estimates Based on BLINEX Loss Function. Based on BLINEX and using equation (24), the Bayes estimation of a parameter $\gamma$ (which can be the scale parameter $\lambda$ or the reliability function $R(t)$ ) is given by

$$
\widehat{\gamma}_{\text {BLINEX }}=-\frac{1}{c} \log \left[\omega_{1} \exp \left[-c \widehat{\gamma}_{\mathrm{ML}}\right]+\omega_{2} E(\exp [-c \gamma] \mid X)\right],
$$

where $\widehat{\gamma}_{\mathrm{ML}}$ is the ML estimate, and $E(\exp [-c \gamma] \mid X)$ can be obtained using

$$
E(\exp [-c \gamma] \mid X)=\int_{0}^{\infty} \exp [-c \gamma] \pi(\lambda \mid X) \mathrm{d} \gamma
$$

Note 1. When $\gamma=\lambda$ in equation (38), the Bayes estimation under BINEX loss function of $\lambda$ and denoted by $\widehat{\gamma}_{\text {BLINEX }}$ is given by

$$
\widehat{\lambda}_{\text {BLINEX }}=-\frac{1}{c} \log \left[\omega_{1} \exp \left[-c \widehat{\lambda}_{\mathrm{ML}}\right]+\omega_{2} E(\exp [-c \lambda] \mid X)\right],
$$

where $\widehat{\lambda}_{\mathrm{ML}}$ is the ML estimate of $\lambda$ and can be obtained using equation (7), and $E(\exp [-c \lambda] \mid X)$ can be obtained using

$$
\begin{aligned}
E(\exp [-c \lambda] \mid X) & =\int_{0}^{\infty} \exp [-c \lambda] \frac{D^{n+\eta}}{\Gamma(n+\eta)} \lambda^{n+\eta-1} \exp [-\lambda D] \mathrm{d} \lambda \\
& =\left(\frac{D}{c+D}\right)^{n+\eta} .
\end{aligned}
$$

Note 2. When $\gamma=R(t)$ in equation (38), then the Bayes estimation under BINEX loss function of $R(t)$, which is denoted by $\widehat{R}_{\text {BLINEX }}(t)$, is given by

$$
\widehat{R}_{\text {BLINEX }}(t)=-\frac{1}{c} \log \left[\omega_{1} \exp \left[-c \widehat{R}_{\mathrm{ML}}(t)\right]+\omega_{2} E\left(\exp \left[-c\left(1-\exp \left[-\lambda t^{-\theta}\right]\right)\right] \mid X\right)\right] \text {, }
$$

where $\widehat{R}_{\mathrm{ML}}(t)$ is the ML estimate of $R(t)$ and can be obtained using equation (8), and $E\left(\exp \left[-c\left(1-\exp \left[-\lambda t^{-\theta}\right]\right)\right] \mid X\right)$ can be obtained using

$$
\begin{aligned}
E\left(\exp \left[-c\left(1-\exp \left[-\lambda t^{-\theta}\right]\right)\right] \mid X\right) & =\int_{0}^{\infty} \exp \left[-c\left(1-\exp \left[-\lambda t^{-\theta}\right] \frac{D^{n+\eta}}{\Gamma(n+\eta)} \lambda^{n+\eta-1} \exp [-\lambda D] \mathrm{d} \lambda\right)\right] \\
& =\left[e^{-c}+e^{-c} D^{n+\eta} \sum_{i=1}^{\infty} \frac{(c)^{i}}{i !}\left(D+i t^{-\theta}\right)^{-(n+\eta)}\right] .
\end{aligned}
$$

Again, we use nonlinear programming to find the optimal values of $\omega_{1}$ and $\omega_{2}$ to compute $\widehat{\lambda}_{\text {BLINEX }}$ and $\widehat{R}_{\text {BLINEX }}(t)$ in equations (41) and (43), respectively. To achieve this target, we minimize the MSE as follows:

$$
\begin{aligned}
\text { Minimize } & \operatorname{MSE}\left(\widehat{\gamma}_{\mathrm{BLINEX}}\right)=E\left(\widehat{\gamma}_{\mathrm{BLINEX}}-\gamma\right)^{2} \\
& =E\left[\left(\omega_{1} \widehat{\gamma}_{\mathrm{ML}}+\omega_{2} E(\exp [-c \gamma] \mid X)-\gamma\right)\right]^{2}
\end{aligned}
$$

subject to $\omega_{1}+\omega_{2}=1,0 \leq \omega_{1}<1,0 \leq \omega_{2}<1$.

\subsubsection{Estimates Based on Weighted Balanced Loss Function.} Based on WBLINEX and using equation (26), the Bayes estimation of a parameter $\gamma$ (which can be the scale parameter $\lambda$ or the reliability function $R(t)$ ) is given by

$$
\widehat{\gamma}_{\text {WBLINEX }}=\frac{1}{c} \log \left[\omega_{1} \exp \left[-c \widehat{\gamma}_{\mathrm{ML}}\right]+\omega_{2} \frac{I_{1}}{I_{2}}\right],
$$

where $\widehat{\gamma}_{\mathrm{ML}}$ is the ML estimate of $\gamma$ and $I_{1}$ and $I_{2}$ can be obtained by

$$
\begin{aligned}
& I_{1}=E(\exp [-z \gamma] \mid X)=\int_{0}^{\infty} \exp [-z \gamma] \pi(\lambda \mid X) \mathrm{d} \lambda \\
& I_{2}=E(\exp [-(c+z) \gamma] \mid X)=\int_{0}^{\infty} \exp [-(c+z)] \pi(\lambda \mid X) \mathrm{d} \lambda .
\end{aligned}
$$

Note 1. When $\gamma=\lambda$ in equation (45), the Bayes estimation under WBINEX loss function of $\lambda$ and denoted by $\widehat{\lambda}_{\text {WBLINEX }}$ is given by

$$
\widehat{\lambda}_{\text {WBLINEX }}=\frac{1}{c} \log \left[\omega_{1} \exp \left[-c \widehat{\lambda}_{\mathrm{ML}}\right]+\omega_{2} \frac{I_{3}}{I_{4}}\right],
$$

where $\widehat{\lambda}_{\mathrm{ML}}$ is the ML estimate of $\lambda$ and can be obtained using equation (7) and $I_{3}$ and $I_{4}$ can be obtained as follows, respectively:

$$
\begin{gathered}
I_{3}=E(\exp [-z \lambda] \mid X)=\int_{0}^{\infty} \exp [-z \lambda] \frac{D^{n+\eta}}{\Gamma(n+\eta)} \lambda^{n+\eta-1} \exp [-\lambda D] \mathrm{d} \lambda=\left(\frac{D}{z+D}\right)^{n+\eta} \\
I_{4}=E(\exp [-(z+c) \lambda] \mid X)=\int_{0}^{\infty} \exp [-(z+c) \lambda] \frac{D^{n+\eta}}{\Gamma(n+\eta)} \lambda^{n+\eta-1} \exp [-\lambda D] \mathrm{d} \lambda=\left(\frac{D}{(z+c)+D}\right)^{n+\eta} .
\end{gathered}
$$


Note 2. When $\gamma=R(t)$ in equation (45), then the Bayes estimation under WBINEX loss function of $R(t)$, which is denoted by $\widehat{R}_{\text {BLINEX }}(t)$, is given by

$$
\widehat{R}_{\text {WBLINEX }}(t)=\frac{1}{c} \log \left[\omega_{1} \exp \left[-c \widehat{R}_{\mathrm{ML}}(t)\right]+\omega_{2} \frac{I_{5}}{I_{6}}\right] \text {, }
$$

where $\widehat{R}_{\mathrm{ML}}(t)$ is the ML estimate of $R(t)$, which can be obtained using equation (8), and $I_{5}$ and $I_{6}$ can be obtained as follows, respectively:

$$
\begin{aligned}
I_{5}=E\left(\exp \left[-z\left(1-\exp \left[-\lambda t^{-\theta}\right]\right)\right] \mid X\right) & =\int_{0}^{\infty} \exp \left[-z\left(1-\exp \left[-\lambda t^{-\theta}\right] \frac{D^{n+\eta}}{\Gamma(n+\eta)} \lambda^{n+\eta-1} \exp [-\lambda D] \mathrm{d} \lambda\right)\right] \\
& =\left[e^{-z}+e^{-z} D^{n+\eta} \sum_{i=1}^{\infty} \frac{(z)^{i}}{i !}\left(D+i t^{-\theta}\right)^{-(n+\eta)}\right], \\
I_{6}=E\left(\exp \left[-(z+c)\left(1-\exp \left[-\lambda t^{-\theta}\right]\right)\right] \mid X\right) & =\int_{0}^{\infty} \exp \left[-(z+c)\left(1-\exp \left[-\lambda t^{-\theta}\right] \frac{D^{n+\eta}}{\Gamma(n+\eta)} \lambda^{n+\eta-1} \exp [-\lambda D] \mathrm{d} \lambda\right)\right] \\
& =\left[e^{-(z+c)}+e^{-(z+c)} D^{n+\eta} \sum_{i=1}^{\infty} \frac{(z+c)^{i}}{i !}\left(D+i t^{-\theta}\right)^{-(n+\eta)}\right] .
\end{aligned}
$$

As in equations (37) and (44), we minimize the MSE to compute $\widehat{\lambda}_{\text {WBLINEX }}$ and $\widehat{R}_{\text {WBLINEX }}(t)$ in equations (47) and (49) as follows, respectively:

$$
\begin{aligned}
& \text { Minimize } \operatorname{MSE}\left(\widehat{\gamma}_{\text {WBLINEX }}\right)=E\left(\widehat{\gamma}_{\text {WBLINEX }}-\gamma\right)^{2}=E\left[\left(\omega_{1} \widehat{\gamma}_{\mathrm{ML}}+\omega_{2} \frac{I_{1}}{I_{2}}-\gamma\right)\right]^{2} \\
& \text { subject to } \omega_{1}+\omega_{2}=1,0 \leq \omega_{1}<1,0 \leq \omega_{2}<1
\end{aligned}
$$

2.4. Simulation Study and Comparisons. In this section, we conduct a Monte Carlo simulation study to compare the performance of the MLE and Bayes estimation under several loss functions, including SE, LINEX, WLINEX, BSE, BLINEX, and BWLINEX to estimate the scale parameter and reliability function of IWD when the shape parameters are known.

Before beginning the simulation, we had to choose some parameters, including $c$ and $z$. We selected the values of $(c)$ as $-0.5,0.5$, and 1 . We selected the positive and negative values to represent both cases of upper estimate and lower estimate, respectively, while the chosen values of $z$ are 3 and -3 .

We conducted the simulation according to the following steps:

(1) For the given values $(\eta=2, v=1)$, we generated a random value $\lambda=1.383$ from the prior PDF as in equation (27).

(2) By using the value $\lambda=1.383$ from Step 1 with selected values of $\theta=3$, we generated data of lower record values from IWD (equation (1) for different sizes $n(n=3,4,5,6)$ and 7$)$.

(3) We computed the estimates of $\lambda, R(t)$ at a chosen time of $t=0.7$ using the estimations under the study.
(4) We repeated Steps 1-3 10,000 times and calculated the MSE for each estimate (say $\varphi$ ) using

$$
\operatorname{MSE}=\frac{1}{10000} \sum_{i=1}^{10000}\left(\widehat{\varphi}_{i}-\varphi\right)^{2}
$$

where $\varphi$ can be $\lambda$ or $R(t)$ and $\widehat{\varphi}_{i}$ is the estimate at the $i^{\text {th }}$ run.

\section{Results and Discussion}

The results of the simulation are listed in Tables 1-4.

In this paper, we employed nonlinear programming to obtain the best values of weighted coefficients $\left(\omega_{1}\right.$ and $\left.\omega_{2}\right)$ of the balanced loss function. The estimates of the parameter $\lambda$ and reliability function $R(t)$ follow the IWD. The estimation methods under study include ML, SE, LINEX, WLINEX, BSE, BINEX, and BWLINEX. We conducted the estimations depending on lower record values.

The main observations of the results are stated in the following points:

(1) Tables 1-4 reveal that the proposed estimator BWLINEX is the absolute best method to estimate 
TABLE 1: MSEs of the estimates of $\lambda$ under unbalanced loss functions at $z=3$.

\begin{tabular}{|c|c|c|c|c|c|c|c|c|}
\hline \multirow{2}{*}{$n$} & \multirow{2}{*}{$\hat{\lambda}_{\mathrm{ML}}$} & \multirow{2}{*}{$\hat{\lambda}_{\mathrm{SE}}$} & \multicolumn{3}{|c|}{$\widehat{\lambda}_{\text {LINEX }}$} & \multicolumn{3}{|c|}{$\widehat{\hat{\lambda}}_{\text {WLINEX }}$} \\
\hline & & & $c=-0.5$ & $c=0.5$ & $c=1$ & $c=-0.5$ & $c=0.5$ & $c=1$ \\
\hline 3 & 4.74099 & 0.67399 & 1.23171 & 0.40932 & 0.27093 & 0.28015 & 0.35097 & 0.38773 \\
\hline 4 & 1.79242 & 0.54569 & 0.89238 & 0.38813 & 0.26938 & 0.23260 & 0.28800 & 0.31639 \\
\hline 5 & 1.12725 & 0.49252 & 0.74656 & 0.33540 & 0.24535 & 0.19347 & 0.23869 & 0.26409 \\
\hline 6 & 0.79418 & 0.42079 & 0.60421 & 0.29610 & 0.23787 & 0.16385 & 0.20625 & 0.22221 \\
\hline 7 & 0.52376 & 0.33702 & 0.46391 & 0.25716 & 0.19196 & 0.13900 & 0.17125 & 0.18800 \\
\hline
\end{tabular}

TABLE 2: MSEs of the estimates of $\lambda$ under balanced loss functions at $z=3$.

\begin{tabular}{ccccccc}
\hline$n$ & $\hat{\lambda}_{\text {BSE }}$ & $c=-0.5$ & $\hat{\lambda}_{\text {BLINEX }}$ & $c=0.5$ & $c=1$ & \multicolumn{3}{c}{$\hat{\lambda}_{\text {WBLINEX }}$} & $c=-0.5$ & $c=0.5$ & $0.3=1$ \\
\hline 3 & 0.65811 & 1.13177 & 0.40829 & 0.27092 & 0.28014 & 0.35097 \\
4 & 0.53206 & 0.81009 & 0.38725 & 0.26938 & 0.23257 & 0.28800 \\
5 & 0.48036 & 0.67664 & 0.33463 & 0.24535 & 0.19300 & 0.23867 \\
6 & 0.40974 & 0.54245 & 0.29541 & 0.23786 & 0.16236 & 0.20606 \\
7 & 0.32708 & 0.41068 & 0.25649 & 0.19196 & 0.13664 & 0.26409 \\
\hline
\end{tabular}

TABLe 3: MSEs of the estimates of $R(t)$ under unbalanced loss functions at $z=-3$.

\begin{tabular}{|c|c|c|c|c|c|c|c|c|}
\hline \multirow{2}{*}{$n$} & \multirow{2}{*}{$\widehat{R}_{\mathrm{ML}}(t)$} & \multirow{2}{*}{$\widehat{R}_{\mathrm{SE}}(t)$} & \multicolumn{3}{|c|}{$\widehat{R}_{\text {LINEX }}(t)$} & \multicolumn{3}{|c|}{$\widehat{R}_{\text {WLINEX }}(t)$} \\
\hline & & & $c=-0.5$ & $c=0.5$ & $c=1$ & $c=-0.5$ & $c=0.5$ & $c=1$ \\
\hline 3 & 0.00301 & 0.00215 & 0.00202 & 0.00229 & 0.00241 & 0.00103 & 0.00113 & 0.00118 \\
\hline 4 & 0.00202 & 0.00168 & 0.00160 & 0.00172 & 0.00188 & 0.00089 & 0.00094 & 0.00102 \\
\hline 5 & 0.00140 & 0.00133 & 0.00127 & 0.00139 & 0.00154 & 0.00076 & 0.00081 & 0.00090 \\
\hline 6 & 0.00091 & 0.00096 & 0.00092 & 0.00104 & 0.00106 & 0.00058 & 0.00065 & 0.00065 \\
\hline 7 & 0.00058 & 0.00068 & 0.00066 & 0.00073 & 0.00076 & 0.00043 & 0.00047 & 0.00049 \\
\hline
\end{tabular}

TABLe 4: MSEs of the estimates of $R(t)$ under balanced loss functions at $z=-3$.

\begin{tabular}{ccccccc}
\hline$n$ & $\widehat{R}_{\mathrm{BSE}}(t)$ & $c=-0.5$ & $\widehat{R}_{\text {BLINEX }}(t)$ & \multicolumn{3}{c}{$\widehat{R}_{\text {BWLINEX }}(t)$} \\
& & $c=0.5$ & $c=1$ & $c=-0.5$ & $c=0.5 \quad 0.00110$ \\
\hline 3 & 0.00191 & 0.00202 & 0.00229 & 0.00241 & 0.00099 & 0.00115 \\
4 & 0.00148 & 0.00160 & 0.00172 & 0.00188 & 0.00085 & 0.00091 \\
5 & 0.00114 & 0.00127 & 0.00139 & 0.00154 & 0.00072 & 0.00078 \\
6 & 0.00078 & 0.00092 & 0.00104 & 0.00106 & 0.00054 & 0.00061 \\
7 & 0.00051 & 0.00065 & 0.00073 & 0.00075 & 0.00039 & 0.00099 \\
\hline
\end{tabular}

scale parameter $\lambda$ and reliability $R(t)$ according to the smallest values of MSEs compared with the estimators of all other methods under study.

(2) For unbalanced loss functions, Tables 1 and 3 showed that the proposed estimator WLINEX is the best method to estimate scale parameter $\lambda$ and reliability $R(t)$ according to the smallest values of MSEs compared with the estimators of ML, SE, and LINEX.

(3) The results show that the values of all MSEs decrease as $n$ increases. This means that there is an inverse relationship between the evaluation functions and the number of recorded values.

(4) It is clear from Table 1 that the ML method has the worst performance in estimating the scale parameter $\lambda$, especially at the small-size samples.

(5) The results show that estimators of the different methods are closer to each other as the sample size increases.
(6) Tables 1 and 2 show that the lower estimate $(c=-0.5)$ of the scale parameter $\lambda$ is better than the upper estimate $(c=0.5$ and $c=1)$ when estimating by WLINEX. However, the upper estimate is better than the lower estimate when estimating by LINEX.

(7) The Bayesian estimators relative to LINEX, WLINEX, and BWLINEX are sensitive to the values of the corresponding shape parameter $c$.

(8) The balanced Bayesian estimator's performance is consistently better than the corresponding unbalanced Bayesian estimator.

\section{Conclusion}

In this work, we developed LINEX to estimate the scale parameter and reliability function of IWD depending on lower record values. The development occurred through merging a weight into LINEX to produce a new loss function called WLINEX. We used WLINEX to derive the scale 
parameter and reliability function of the IWD. The majority of earlier researchers have determined the weighted balanced coefficients by arbitrarily providing the summation equal to one. In this work, we depended on justifiable mathematical methods to determine these coefficients, where we utilized nonlinear programming to obtain the optimal values of the weighted coefficients for each of BSE, BLINEX, and BWLINEX.

Furthermore, we conducted a Monte Carlo simulation to examine the performance of the proposed methods: WLINEX and BWLINEX. We then compared the proposed methods with the other methods, including ML, SE, LINEX, BSE, and BLINEX. The results of the simulation showed that the developed estimators in this work (BWLINEX and WLINEX) have the best performance in estimating scale parameter and in estimating reliability according to the smallest values of MSE, respectively. This result shows that the approach followed is promising and can be applied in a real environment.

\section{Data Availability}

The data were generated by simulation done by mathematical software. The simulation is in the Supplementary materials.

\section{Conflicts of Interest}

The author declares that he has no conflicts of interest.

\section{Acknowledgments}

This project was supported by the Deanship of Scientific Research at Prince Sattam bin Abdulaziz University under the research project no. 2020/01/12030.

\section{Supplementary Materials}

The contribution of this paper depends on two parts including theoretical part and empirical part. The data in empirical part were generated by Monte Carlo simulation. (Supplementary Materials)

\section{References}

[1] N. Balakrishnan, P. S. Chan, and M. Ahsanullah, "Recurrence relations for moments of record values from generalized extreme value distribution," Communications in Statistics-Theory and Methods, vol. 22, no. 5, pp. 1471-1482, 1993.

[2] P. Nasiri, \&, and S. Hosseini, "Statistical inferences for Lomax distribution based on record values (bayesian and classical)," Journal of Modern Applied Statistical Methods, vol. 11, no. 1, p. 15, 2012.

[3] F. Al-Duais and M. Alhagyan, "Bayesian estimates based on record values under weighted LINEX loss function," Pakistan Journal of Statistics and Operation Research, vol. 16, pp. 11-19, 2020.

[4] F. Yang, H. Ren, and Z. Hu, "Maximum likelihood estimation for three-parameter Weibull distribution using evolutionary strategy," Mathematical Problems in Engineering, vol. 2019, pp. p-8, Article ID 6281781, 2019.
[5] B. X. Wang and Z.-S. Ye, "Inference on the Weibull distribution based on record values," Computational Statistics \& Data Analysis, vol. 83, pp. 26-36, 2015.

[6] S. Singh, Y. Mani Tripathi, and S.-J. Wu, "Bayesian estimation and prediction based on lognormal record values," Journal of Applied Statistics, vol. 44, no. 5, pp. 916-940, 2017.

[7] B. Al-Zahrani, S. Shahbaz, \&, and M. Shahbaz, "Record values of ratio of Weibull random variables," Journal of the National Science Foundation of Sri Lanka, vol. 46, no. 2, 2018.

[8] A. Pak and S. Dey, "Statistical inference for the power Lindley model based on record values and inter-record times," Journal of Computational and Applied Mathematics, vol. 347, pp. 156-172, 2019.

[9] H. Piriaei, G. Yari, and R. Farnoosh, "E-Bayesian estimations for the cumulative hazard rate and mean residual life based on exponential distribution and record data," Journal of Statistical Computation and Simulation, vol. 90, no. 2, pp. 271-290, 2020.

[10] A. Pak, A. A. Jafari, \&, and N. B. Khoolenjani, "On reliability in a multicomponent stress-strength generalized Rayleigh model based on record values," Journal of Testing and Evaluation, vol. 48, no. 6, 2020.

[11] J. Cai, Y. Shi, and Y. Zhang, "Robust Bayesian analysis for parallel system with masked data under inverse Weibull lifetime distribution," Communications in Statistics - Theory and Methods, vol. 49, no. 6, pp. 1422-1434, 2020.

[12] M. M. M. El-Din, F. H. Riad, and M. A. El-Sayed, "Statistical inference and prediction for the inverse Weibull distribution based on record data," Journal of Statistics Applications \& Probability, vol. 3, no. 2, pp. 171-177, 2014.

[13] M. Y. Lee, "On characterizations of the inverse Weibull distribution based on record values," Journal of Applied Mathematics \& Informatics, vol. 36, no. 5-6, pp. 429-433, 2018.

[14] A. K. Mbah, \&, and C. P. Tsokos, "Record values from half logistics and inverse Weibull probability distribution functions," Neural Parallel and Scientific Computations, vol. 16, no. 1, p. 73, 2008.

[15] K. S. Sultan, "Record values from the inverse Weibull lifetime model: different methods of estimation," Intelligent Information Management, vol. 2, no. 11, pp. 631-636, 2010.

[16] M. Tayyab, K. Khan, and A. Ahmad, "Analysis of inverse Weibull distribution based on record values," Journal of Statistics Applications \& Probability, vol. 2, no. 3, p. 281, 2013.

[17] R. Calabria and G. Pulcini, "Point estimation under asymmetric loss functions for left-truncated exponential samples," Communications in Statistics - Theory and Methods, vol. 25, no. 3, pp. 585-600, 1996.

[18] G. Gencer, \&, and B. Saraçoğlu, "Comparison of approximate Bayes estimators under different loss functions for parameters of odd Weibull distribution," Journal of Selçuk University Natural and Applied Science, vol. 5, no. 1, pp. 18-32, 2016.

[19] N. Khatun and M. A. Matin, "A study on LINEX loss function with different estimating methods," Open Journal of Statistics, vol. 10, no. 01, p. 52, 2020.

[20] A. Parsian, \&, and S. N. U. A. Kirmani, "Estimation under LINEX loss function," in Handbook of Applied Econometrics and Statistical Inference, A. Ullah, A. T. K. Wan, and A. Chaturvedi, Eds., CRC Press, Boca Raton, FL, USA, 2002.

[21] D. R. Barot and M. N. Patel, "Posterior analysis of the compound Rayleigh distribution under balanced loss functions for censored data," Communications in Statistics-Theory and Methods, vol. 46, no. 3, pp. 1317-1336, 2017. 
[22] A. H. Abd Ellah, "Bayesian and non-Bayesian estimation of the inverse Weibull model based on generalized order statistics," Intelligent Information Management, vol. 4, no. 2, pp. 23-31, 2012.

[23] Z. F. Jaheen and M. M. Al Harbi, "Bayesian estimation for the exponentiated Weibull model via Markov chain Monte Carlo simulation," Communications in Statistics-Simulation and Computation, vol. 40, no. 4, pp. 532-543, 2011.

[24] K. S. Sultan, "Bayesian estimates based on record values from the inverse Weibull lifetime model," Quality Technology \& Quantitative Management, vol. 5, no. 4, pp. 363-374, 2008.

[25] H. R. Varian, "A Bayesian approach to real estate assessment," in Studies in Bayesian Econometric and Statistics in Honor of Leonard J. Savage, North-olland Pub. Co., North-Holland, Amsterdam, 195-208, 1975. 